\title{
Clinical Outcomes of Drug-Coated Balloons in Coronary Artery Disease Unsuitable for Drug-Eluting Stent Implantation
}

\author{
Raisuke Iijima, MD, PhD; Norihiro Kougame, MD; Hidehiko Hara, MD, PhD; \\ Masao Moroi, MD, PhD; Masato Nakamura, MD, PhD
}

\begin{abstract}
Background: The aim of this study was to investigate whether drug-coated balloon (DCB) treatment is effective for de novo coronary lesions that are unsuitable for drug-eluting stent (DES) implantation.

Methods and Results: This retrospective study included 118 de novo lesions that were not suitable for DES implantation. Of the lesions, $40 \%$ was treated because of very small vessel disease. Patients with planned non-cardiac surgery and at high bleeding risk were $3 \%$ and $19 \%$, respectively, and lesions that easily develop stent fracture comprised $26 \%$. Clinically driven target lesion revascularization (TLR) was the primary endpoint. The rate of suboptimal lesion preparation before DCB treatment was set as the secondary endpoint. Optimal lesion preparation was defined as acquisition of Thrombolysis in Myocardial Infarction flow grade 3 , minor coronary dissection, and residual stenosis $\leq 30 \%$. The rate of suboptimal lesion preparation was $2.5 \%$ and 3 patients needed bail-out stenting. Accordingly, 115 patients were treated with a DCB. Clinically driven TLR had occurred in 8 patients $(7.0 \%)$ at the 8-month follow-up. The presence of chronic total occlusion was identified as an independent predictor for TLR (odds 11.57; 95\% confidence interval, 1.38-135.54; $P=0.02)$.
\end{abstract}

Conclusions: For lesions that are unsuitable for stent implantation, stent-less intervention using a DCB should be considered initially. The present study also highlighted that lesion preparation is key to a successful DCB strategy.

Key Words: Drug-coated balloons; Drug-eluting stents; Lesion preparation; Small coronary vessel disease; Stent fractures

$\mathbf{M}$ any studies have demonstrated that in coronary artery disease (CAD) patients, drug-eluting stents (DES) dramatically reduce clinical restenosis compared with bare metal stents. ${ }^{1}$ Consequently, DES play a central role in percutaneous coronary intervention (PCI) procedures in patients with significantly narrowed coronary vessels. On the other hand, it is also a fact that some target coronary lesions are unsuitable for DES implantation. ${ }^{2-4}$ For example, in patients with very small vessel coronary disease, patients planned for non-cardiac surgery, and in lesions that easily develop stent fractures, implantation of any metal in the coronary vessel is best avoided. We have experienced that these subsets are potentially troublesome in clinical practice. The clinician must coordinate with individual patients in terms of the duration of antiplatelet therapy prior to the day of surgery. Also, the risk of repeated target revascularization related to stent fracture is increased in the event of stent thrombosis.

In recent years, drug-coated balloon (DCB) therapy has been rapidly accepted in clinical practice, especially for the treatment of in-stent restenosis. ${ }^{\mathbf{5 , 6}}$ More recently, the use of
DCB is spreading to de novo CAD. ${ }^{7}$ The DCB has beneficial features, such as allowing local drug delivery without the need to implant any metal. Subsequently, the duration of dual antiplatelet therapy (DAPT) is shortened. Importantly, the DCB is just a delivery vehicle, and does not assist in dilating narrowed coronary vessels. ${ }^{5}$ Thus, lesion preparation with standard balloon angioplasty is the key to successful DCB treatment, and is essential before the use of a DCB. The aim of effective lesion preparation is to gain sufficient luminal enlargement, while minimizing coronary dissection. In the present study, we investigated whether DCB therapy was effective for de novo CAD unsuitable for DES implantation. Also, we present our clinical experiences with regard to plaque modification of the target lesion before DCB dilation.

\section{Methods}

\section{Study Population}

The aim of this retrospective study was to investigate the effectiveness of DCB in de novo CAD that was unsuitable

Received January 25, 2018; revised manuscript received April 9, 2018; accepted May 6, 2018; released online June 12 , 2018 Time for primary review: 26 days

Toho University School of Medicine, Ohashi Medical Center, Tokyo, Japan

The first two authors contributed equally to this work (R.I., N.K.).

Mailing address: Raisuke Iijima, MD, Toho University School of Medicine, Ohashi Medical Center, 2-17-6 Ohashi, Meguro-ku, Tokyo 153-8515, Japan. E-mail: raisuke@live.jp

ISSN-1346-9843 All rights are reserved to the Japanese Circulation Society. For permissions, please e-mail: cj@j-circ.or.jp 


\begin{tabular}{|c|c|}
\hline No. of patients & 118 \\
\hline Age, years & $68.2 \pm 12.2$ \\
\hline Male & $101(86)$ \\
\hline Hypertension & $90(76)$ \\
\hline Dyslipidemia & $74(63)$ \\
\hline Current smoker & $59(50)$ \\
\hline Diabetes mellitus & $66(56)$ \\
\hline \multicolumn{2}{|l|}{ Clinical presentation } \\
\hline Symptomatic stable angina & $59(50)$ \\
\hline Asymptomatic stable angina & $34(29)$ \\
\hline Unstable angina pectoris & $25(21)$ \\
\hline Killip classification & $1.0 \pm 0.4$ \\
\hline Prior Ml & $41(35)$ \\
\hline Prior coronary intervention & $72(61)$ \\
\hline Prior coronary aortic bypass & $6(5)$ \\
\hline Chronic kidney disease & $46(39)$ \\
\hline Hemodialysis & $30(25)$ \\
\hline eGFR, $\mathrm{mL} / \mathrm{min} / 1.73 \mathrm{~m}^{2}$ & $46.8 \pm 28.1$ \\
\hline LVEF, \% & $56.1 \pm 14.9$ \\
\hline Multivessel disease & $68(58)$ \\
\hline \multicolumn{2}{|l|}{ Reasons for DCB treatment } \\
\hline Small coronary vessel disease & $47(40)$ \\
\hline Risk of stent fracture & $31(26)$ \\
\hline Risk of bleeding complications & $23(19)$ \\
\hline Lesion involving side branches & $13(11)$ \\
\hline Planned non-cardiac surgery & $4(3)$ \\
\hline
\end{tabular}

Data are presented as $n(\%)$ or mean \pm SD. DCB, drug-coated balloon; eGFR, estimated glomerular filtration rate; LVEF, left ventricular ejection fraction; MI, myocardial infarction.

for DES implantation. The following conditions were considered as unsuitable de novo CAD cases: (1) very small vessel coronary disease; target vessel diameter $\leq 2.0$ $\mathrm{mm}$ by visual estimate on angiograms; (2) patients planned for non-cardiac surgery or are at high risk of bleeding; (3) lesions that easily develop stent fractures; and (4) $>50 \%$ diameter stenosis in the contiguous side branch in true bifurcated lesions. Clinical and procedural data were entered into a database beforehand and analyzed retrospectively. Written informed consent was given by all patients.

\section{Lesion Preparation and DCB Procedure}

Unlike metal stent implantation, the DCB strategy has 2 steps. First, lesion preparation. For this purpose, we used a standard semicompliant balloon or the Lacrosse ${ }^{\circledR}$ non-slip element (NSE) balloon (Goodman Co., Ltd., Nagoya, Japan), which is an angioplasty catheter with 3 longitudinal elements attached directly proximal and distal to the balloon, which produce 3 endovascular surgical incisions during balloon dilation. ${ }^{8}$ To avoid major coronary dissection, the initial balloon size of the NSE was visually estimated as one-quarter less than the vessel diameter. Balloon inflation pressure ranged from 6 to 14 atmospheres (atm), and multiple inflations were done. The paclitaxelcoated $\left(3 \mu \mathrm{g} / \mathrm{mm}^{2}\right)$ balloon catheter based on Paccocath ${ }^{\circledR}$ Technology (SeQuent ${ }^{\circledR}$ Please, B. BraunMelsungen AG) was used in the present study. ${ }^{7}$ After successful lesion preparation, we used a DCB of the same size as the predilatation balloon or one-quarter larger than it. The recom-

\begin{tabular}{|lc|}
\hline \multicolumn{2}{|c|}{ Table 2. Baseline Characteristics of Lesions and } \\
Procedures \\
No. of lesions \\
Lesion characteristics \\
Target lesion \\
Left anterior descending \\
Left circumflex \\
Right coronary artery \\
Pre TIMI flow grade \\
Post TIMI flow grade & $32(27)$ \\
Type B2/C & $52(44)$ \\
Severe angulation & $34(29)$ \\
Angle difference & $2.2 \pm 1.2$ \\
Hinge motion & $2.9 \pm 0.3$ \\
Bifurcation & $63(53)$ \\
Severe calcification & $28(24)$ \\
Chronic total occlusion & $25.8 \pm 27.6$ \\
Eccentric & $21(18)$ \\
Procedural characteristics & $51(43)$ \\
Transradial intervention & $34(29)$ \\
Any imaging device used & $17(14)$ \\
IVUS & $33(28)$ \\
OFDI & \\
Lesion preparation before DCB & \\
Rotablator & \\
Lacrosse NSE balloon ${ }^{\circledR}$ & $17(67)$ \\
Conventional balloon/cutting balloon & $110(93)$ \\
Final balloon size, mm & $98(83)$ \\
Balloon/artery ratio & $12(10)$ \\
Dilation pressure, atm & \\
DCB & $12(10)$ \\
No. of DCB used & $76(66)$ \\
Balloon diameter, mm & $39(34)$ \\
\hline
\end{tabular}

Data are presented as $\mathrm{n}(\%)$ or mean $\pm \mathrm{SD}$. DCB, drug-coated balloon; IVUS, intravascular ultrasound; NSE, non-slip element; OFDI, optical frequency domain imaging; TIMI, Thrombolysis in Myocardial Infarction.

mended inflation time is $1 \mathrm{~min}$ for a maximum balloon pressure of $10 \mathrm{~atm}$. Regarding antiplatelet therapy, patients scheduled for PCI were administered acetylsalicylic acid $(100 \mathrm{mg})$ and clopidogrel $(75 \mathrm{mg})$ or prasugrel $(3.5 \mathrm{mg})$ daily for at least 3 days before the procedure. In addition, all patients received DAPT for 1-3 months after the procedure. ${ }^{5}$ Continuation of DAPT was at physician's discretion.

\section{Study Endpoints and Definitions}

Clinically driven target lesion revascularization (TLR) and angiographic binary restenosis were the primary endpoints of the study. The chronic-phase evaluations were scheduled at 8 months after PCI. The rate of suboptimal lesion preparation before DCB treatment was set as the secondary endpoint. The following 3 criteria were considered necessary for optimal lesion preparation: (1) normal coronary flow after predilatation (Thrombolysis in Myocardial Infarction [TIMI] flow grade 3); (2) none or only minor dissections (class $\mathrm{A}-\mathrm{C}$ according to the classification of the North American National Heart Lung and Blood Institute 
(A) Coronary dissection

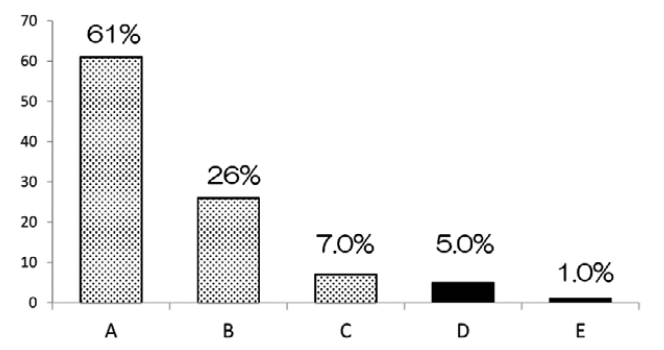

(B) Residual stenosis

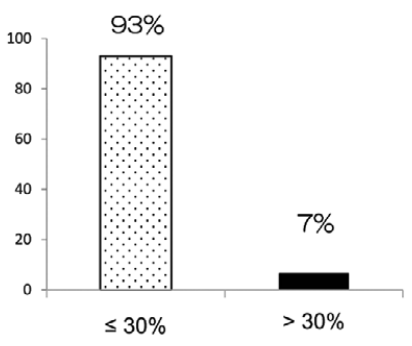

(C) TIMl flow grade

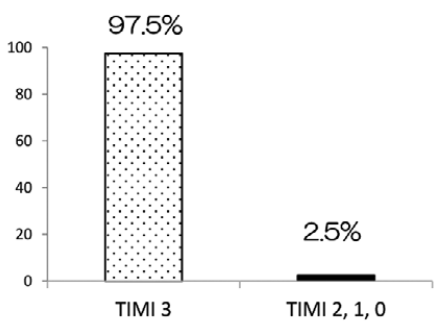

(D) Lesion preparation after balloon angioplasty

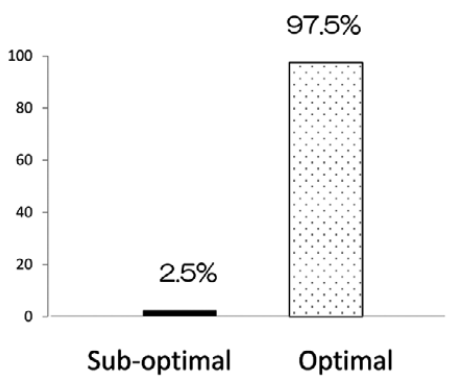

Figure 1. (A) Distribution of coronary dissection after lesion preparation. Coronary dissections were evaluated using the NHLBI classification A-E. (B) Incidence of residual stenosis $<30 \%$ after lesion preparation. (C) Incidence of TIMI flow grade $<3$ after lesion preparation. (D) Percentage of lesions with suboptimal lesion preparation (Left) and optimal lesion preparation (Right). NHLBI, National Heart, Lung, and Blood Institute; TIMI, Thrombolysis in Myocardial Infarction.

[NHLBI]); and (3) residual stenosis $\leq 40 \%$ according to quantitative analysis as visual stenosis $\leq 30 \%$, because visual distinction of a stenosis between $30 \%$ and $40 \%$ is often impossible. ${ }^{910}$ If all 3 criteria were not achieved after standard balloon angioplasty, it was defined as "suboptimal lesion preparation". For example, a lesion with residual stenosis $>40 \%$ with a type $\mathrm{D}$ dissection and delayed distal flow.

Abrupt coronary vessel closure was considered to have occurred when angiography confirmed the presence of TIMI flow grade 0 or I, or when flow-limiting thrombus occurred at any time after the DCB procedure. TLR was considered clinically indicated if the angiographic percentage diameter stenosis of the then-treated lesion was $\geq 50 \%$ in the presence of ischemic signs or symptoms or if the diameter stenosis was $\geq 70 \%$, irrespective of ischemic signs or symptoms. ${ }^{11}$ The diagnosis of myocardial infarction (MI) required the presence of new Q-waves on ECG and/ or a creatine kinase-MB isoenzyme level (or total creatine kinase if creatine kinase-MB was not available) twice the upper limit of the normal range. ${ }^{12}$ Many studies have reported the angiographic and procedural predictors of the circumstances under which a stent fracture is relatively easy to occur., ${ }^{3,13,14}$ Abnormal movement caused by torsion or flexion forces during the cardiac cycle is considered a marker of lesions that easily develop stent fracture. Therefore, target lesions that readily cause stent fracture were defined as follows: (1) target lesion requiring multiple stenting in the proximal to mid segment of the right coronary artery; (2) presence of extreme angulation $\left(>90^{\circ}\right)$ proximal to or within the target lesion; and (3) lesion with a hinge motion having $\geq 16^{\circ}$ difference in the angle between diastole and systole on coronary angiography. ${ }^{13,14}$ Noncardiac surgery was defined as any procedure performed under general, spinal, or local anesthesia. ${ }^{15}$ High bleeding risk was defined as a history of major bleeding or taking any anticoagulant drugs. ${ }^{4}$ Quantitative coronary angiography was performed before lesion preparation, during lesion preparation, after predilatation, and immediately after the procedure using edge detection algorithms. Minimum lumen diameter (MLD), reference vessel diameter, diameter stenosis, and lesion length were measured using a single, matched worst view. Qualitative morphological lesion characteristics were characterized by standard criteria. ${ }^{16,17}$

\section{Statistical Analysis}

The data are presented as mean \pm SD or counts $(\%)$. Comparisons between groups were performed by paired Student's t-test for continuous variables. A multivariate logistic regression model was used to determine the predictors of clinically driven TLR. All variables are summarized in Table $\mathbf{1}$ and Table 2, and quantitative coronary angiography findings were entered into the models. The odds ratio (OR) and $95 \%$ confidence intervals $(\mathrm{CI})$ for clinically relevant variables with $\mathrm{P}<0.05$ following univariate analyses were included in the final multivariate model. All analyses were carried out with the SAS statistics package (JMP ${ }^{\circledR} 11$; SAS Institute Inc., Cary, NC, USA). 


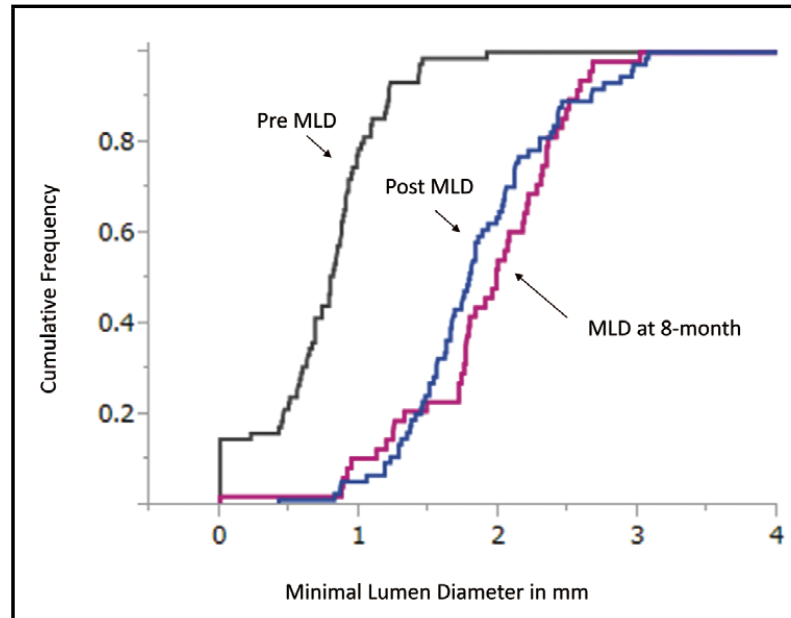

Figure 2. Cumulative distribution function curves for minimum lumen diameter (MLD) before, immediately after and at 8 months after percutaneous coronary intervention.

\section{Results}

\section{Baseline Characteristics of the Patients}

Table 1 shows the baseline clinical and angiographic characteristics of the patients. Since May 2014 we enrolled 118 patients, of whom 93 had stable angina pectoris with proven myocardial ischemia, as evidenced by echocardiography $(15 \%)$ and stress tests with ECG and scintigraphy $(76 \%)$, or fractional flow reserve during coronary angiography $(9 \%)$. The remaining 25 patients were hospitalized for an episode of unstable angina pectoris (symptom lasting $<48 \mathrm{~h}$ ) according to the Braunwald classification. The reasons for choosing treatment using a DCB were as follows: very small vessel coronary disease in 47 lesions $(40 \%)$; patients scheduled for non-cardiac surgery or with high risk of bleeding complications (3\% and $19 \%$, respectively); 31 lesions were found in target narrow vessels considered to easily develop stent fracture (26\%). Regarding side branches, a DCB was used in cases of coronary stenosis in which main CAD was treated with DES. The DCB was used more frequently in the left circumflex coronary artery.
Chronically occluded and severely calcified lesions comprised $14 \%$ and $29 \%$, respectively. Hinge motion with $\geq 16^{\circ}$ difference in angles was also observed in 21 patients by coronary angiography.

\section{Lesion Preparation and Clinical Restenosis}

Most procedures were performed under imaging guidance (Table 2). Major coronary dissection (NHLBI classes D and E) and inadequate lumen enlargement after standard balloon angioplasty were observed in $6 \%$ and $7 \%$ of cases, respectively (Figure 1A,B). In addition, 3 lesions $(2.5 \%)$ did not achieve TIMI flow grade 3 at the time of lesion preparation (Figure 1C). Subsequently, 3 lesions (2.5\%) failed to achieve correct lesion preparation, defined according to the 3 criteria previously described (Figure 1D). These 3 patients with suboptimal lesion preparation required stent implantation as bail-out for type $\mathrm{E}$ dissections with delayed distal flow. Accordingly, 115 patients were solely treated with a DCB. Regarding the device used for predilatation, the NSE balloon was used in $66 \%$ of lesions, with a mean size of $2.52 \pm 0.53 \mathrm{~mm}$ and pressure of $13.3 \pm 3.1 \mathrm{~atm}$. Subsequently, the ratio of balloon to artery was 1.15 . When the efficacy of the NES for lesion preparation was compared with other devices, the incidence of major dissection was significantly lower in target lesions treated with the NSE $(2.6 \%)$ than with other devices $(12.5 \%, \mathrm{P}=0.045)$, and the percent diameter stenosis reflecting residual stenosis was significantly lower in the NES group than in the other device groups $(18.8 \pm 9.7$ vs. $25.6 \pm 15.0, \mathrm{P}=0.004)$. However, there was no difference in the acquisition of TIMI 3 grade flow between the 2 groups (97.4\% vs. $97.5 \%, \mathrm{P}=0.97)$. Abrupt coronary vessel closure and MI were not observed during the 8 months of follow-up among the patients with DCB treatment. There was observed 1 cardiac and 2 noncardiac deaths during the follow-up period.

Figure 2 shows the cumulative distribution function curve for the MLD before, immediately after, and at 8 months after PCI. The curves for 8 months after PCI slightly shifted to right of those for immediately after PCI, indicating late luminal enlargement of the treated vessel after PCI with a DCB. At the 8-month follow-up time point, clinical and angiographic follow-up rates were $98 \%$ and $66 \%$, respectively (Figure 3A). Clinically driven TLR occurred in 8 patients $(7.0 \%)$ at 12 months after the procedure. Five patients had obvious ischemic symptoms and remaining 3
(A)

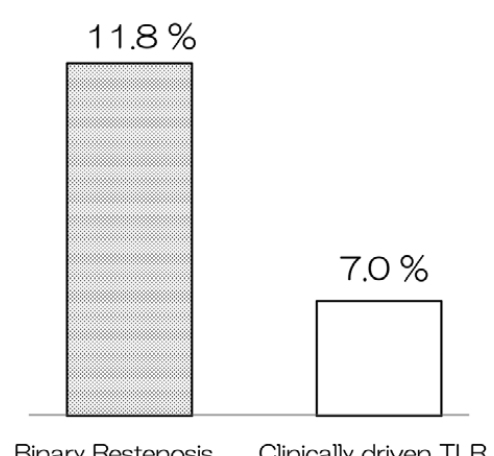

(B)

TLR according to vessel size

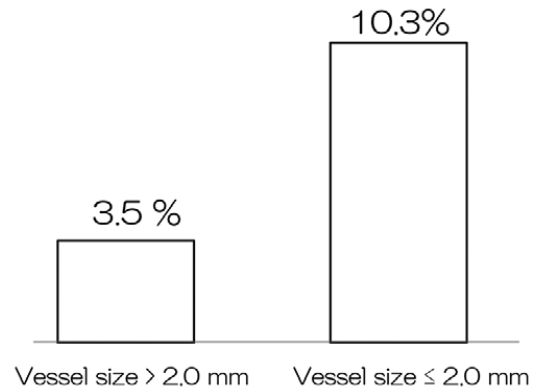

Figure 3. (A) Incidence of binary restenosis and target lesion revascularization (TLR). (B) Clinically driven TLR according to vessel size. 


\begin{tabular}{|c|c|c|c|}
\hline & $\begin{array}{c}\text { TLR (+) } \\
(n=8)\end{array}$ & $\begin{array}{l}\text { TLR (-) } \\
(n=107)\end{array}$ & $P$ value \\
\hline \multicolumn{4}{|l|}{ Before PCI } \\
\hline Reference vessel diameter, $\mathrm{mm}$ & $1.86 \pm 0.21$ & $2.20 \pm 0.56$ & 0.09 \\
\hline MLD, mm & $0.43 \pm 0.36$ & $0.80 \pm 0.44$ & 0.03 \\
\hline Diameter stenosis, \% & $78.3 \pm 18.3$ & $64.6 \pm 16.3$ & 0.03 \\
\hline Lesion length, $\mathrm{mm}$ & $22.4 \pm 6.9$ & $12.4 \pm 9.2$ & 0.004 \\
\hline \multicolumn{4}{|l|}{ Post PCI } \\
\hline Reference vessel diameter, $\mathrm{mm}$ & $2.05 \pm 0.14$ & $2.37 \pm 0.57$ & 0.12 \\
\hline MLD, mm & $1.50 \pm 0.25$ & $1.88 \pm 0.60$ & 0.08 \\
\hline Diameter stenosis, \% & $27.1 \pm 10.1$ & $21.5 \pm 13.3$ & 0.25 \\
\hline Balloon size, $\mathrm{mm}$ & $2.33 \pm 0.36$ & $2.42 \pm 0.54$ & 0.71 \\
\hline
\end{tabular}

Data are presented as number of patients $(\%)$ or mean \pm SD. MLD, minimal luminal diameter; $\mathrm{PCl}$, percutaneous coronary intervention.

\begin{tabular}{|c|c|c|c|c|}
\hline \multirow{2}{*}{ Variables } & \multicolumn{2}{|c|}{ Univariate } & \multicolumn{2}{|c|}{ Multivariate } \\
\hline & OR $[95 \% \mathrm{Cl}]$ & P value* & OR $[95 \% \mathrm{Cl}]$ & P value \\
\hline Chronic total occlusion & 33.67 [6.71-254.65] & $<0.001$ & $11.57[1.38-135.54]$ & 0.02 \\
\hline Vessel size (1.0 mm decrease) & $4.68[1.0-29.26]$ & 0.050 & & \\
\hline Post MLD (0.1 mm decrease) & $3.81[0.92-20.63]$ & 0.070 & & \\
\hline Lesion length (1.0 $\mathrm{mm}$ decrease) & $0.92[0.85-0.98]$ & 0.01 & & \\
\hline
\end{tabular}

*Univariate analysis, "multivariate analysis. $\mathrm{Cl}$, confidence interval; MLD, minimum lumen diameter; OR, odds ratio.

asymptomatic patients showed significant lumen narrowing with $>90 \%$ diameter stenosis. There were no significant differences in terms of baseline clinical characteristics between patients with and without TLR (Table S1). However, in patients with TLR, the presence of chronic total occlusion (CTO) was significantly higher and a much smaller DCB was used (Table S2). Table 3 shows quantitative coronary angiography data for before and immediately after PCI. Patients with TLR tended to have smaller vessel size and significantly smaller MLD before the procedure. The MLD after PCI also tended to be smaller in patients with clinically driven TLR. Among the patients with very small vessel coronary disease with a reference diameter $\leq 2.0 \mathrm{~mm}$ on quantitative analysis (Figure 3B), clinically driven TLR occurred in $6(10.3 \%)$.

Logistic regression analysis was used to determine independent predictors of clinically driven TLR: CTO, vessel size, post MLD, and lesion length were identified. Finally, the presence of CTO was identified as an independent predictor for clinically driven TLR (Table 4).

\section{Discussion}

The present study showed that as long as optimal lesion preparation is achieved, DCB angioplasty is an effective strategy for de novo CAD, especially in lesions that are unsuitable for DES implantation. The main findings are summarized as follows: the rate of clinically driven TLR was $7.0 \%$ in consecutive de novo CAD with lesions that were unsuitable for DES implantation. Furthermore, MI, and abrupt coronary vessel closure did not occur within 8 months of PCI with a DCB. A strong association was found between clinical restenosis and CTO lesions.
The DCB has the beneficial feature of delivering an adequate dose of paclitaxel locally to the target lesion without the implantation of any metal. Therefore, it can be viewed as a breakthrough device, especially in the treatment of recurrent restenosis after treatment with metal stents. ${ }^{5}$ Accordingly, treatment with DCB is now being studied as a stand-alone treatment for de novo CAD.6,7 Although some studies have reported promising outcomes for DCB procedures, we consider that it is not a complete substitute for a DES strategy in all cases of de novo CAD, in terms of preventing abrupt vessel closure after coronary artery dilation or reducing the incidence of repeat revascularization in the chronic phase. ${ }^{1}$ Therefore, we focused on target lesions that were unsuitable for DES implantation, which is often the case in clinical practice. In the present study, the most common reason for choosing the DCB strategy was for the treatment of very small coronary vessel disease for which implantation of a metal stent of size $2.25 \mathrm{~mm}$ was not possible. The second most common reason was to avoid the risk of stent fracture. Stent fracture commonly develops in specific culprit lesions with more torsion and excessive movement during the cardiac cycle, such as ostial lesions of the right or left circumflex coronary artery and calcified lesions with a hinge point. , $^{3,13,14}$ In addition, $15 \%$ of patients treated with a coronary intervention are at a high risk of bleeding complications, and the proportion of patients who require non-cardiac surgery is increasing by 4\% each year. ${ }^{15,18}$ Assuming that an involuntary interruption of antiplatelet therapy might occur in patients treated with PCI, the DCB treatment is expected to have advantages, especially in the subset focused on in the present study. 


\section{Clinical Restenosis}

Recent clinical studies demonstrated a rate of TLR with new-generation DES of approximately $5 \%$ at 12 months. ${ }^{19,20}$ Clinically driven TLR was 7\% in the present study, which should be interpreted carefully because we enrolled culprit lesions with smaller vessel size, CTO lesions, and patients with hemorrhagic risk, which are cases that are usually excluded in randomized control trials. ${ }^{19,20}$ Also, we used the DCB for lesions considered to be at risk for the development of stent fracture based on the angiographic findings. Previous studies have demonstrated that stent fracture is significantly associated with an increased risk of major adverse cardiac events within 9 months; in particular, a TLR rate of $25.6 \%$ was quite high in the era of newgeneration DES. ${ }^{21,22}$ Therefore, it may be better to avoid routine stent implantation in lesions with a hinge motion, tortuosity, and severe calcification. The present study found that achieving optimal lesion preparation with a debulking device and NSE balloon were key to a low incidence of TLR in such lesions. Another important finding was that lesion morphology and severity were involved with the incidence of clinical restenosis. Indeed, plaque morphology at the target lesion is an important determinant of clinical outcomes after balloon angioplasty. ${ }^{23}$ The present study showed that CTO was an independent predictor of clinically driven TLR: a DCB was used in 17 CTO lesions; (10 lesions of very small coronary vessel disease, 4 patients with high bleeding risk, and 3 lesions with a risk of stent fracture). There reasons for choosing a DCB to treat these lesions were to avoid the use of multiple stenting, and to enlarge a narrowing distal vessel without stenting in the chronic phase. However, complex lesions with high plaque volumes such as CTO may be limitation to the use of DCB.

\section{Lesion Preparation Before DCB}

The major concern with the DCB procedure is abrupt coronary vessel closure that may occur as a result of varying degrees of vessel wall injury, ranging from slight intimal tear to considerable medial dissection. ${ }^{24}$ The DCB is merely a vehicle for elution of antiproliferative drugs, and not an angioplasty tool. 5 Therefore, the key to successful DCB treatment depends on whether lesion preparation is appropriately performed before dilation of the balloon. In other words, the strategy should be changed from DCB to metal stent implantation if lesion preparation does not meet all 3 of the previously described criteria; that is, if residual stenosis $>30 \%$ or major dissection with delayed distal flow are observed. Consequently, all of the present patients treated with a DCB after optimal lesion preparation did not develop MI and abrupt coronary vessel closure within 8 months of the procedure. This finding could dispel the perception that PCI with balloon angioplasty has a risk of abrupt coronary vessel closure. One of the reasons why DCB angioplasty can be performed safely is the recent development of antiplatelet therapy, which makes it more effective and reliable than balloon angioplasty used in the past..$^{25}$ In the present study, prompt treatment with reliable antiplatelet drugs such as prasugrel or clopidogrel may have contributed to the prevention of abrupt coronary vessel closure. Furthermore, the present study showed that the use of an NSE balloon was effective for optimal lesion preparation before the use of the DCB. The use of an NSE balloon was associated with a lower incidence of major coronary dissection and residual stenosis, although it depends on operator decision. An NSE balloon can reduce elastic recoil, traumatic vessel injury, and dissection, because of the 3 endovascular surgical incisions made during balloon dilation. ${ }^{8}$ Therefore, an NSE balloon may be useful as a tool for lesion preparation before the use of the DCB.

\section{Study Limitations}

Before drawing conclusions, the findings of the present study should be carefully interpreted, because this was a single-center study with a small sample size. The low rate of follow-up angiography was also a major limitation. However, it might reflect recent Japanese clinical practice. The recently published ReACT trial showed that routine angiographic follow-up did not provide any clinical benefits as compared with clinical follow-up. ${ }^{26}$ The rate of clinical follow-up was $98 \%$ in the present study, so we consider there were very few asymptomatic patients with myocardial ischemia. Furthermore, we did not compare the efficacy of balloon angioplasty or $2.25-\mathrm{mm}$ DES, because of having a single-arm protocol. The study population comprised patients or lesions that were unsuitable for DES implantation, but there is no consensus regarding the suitability of DES implantation. There is room for discussion when it comes to lesions that easily develop stent fracture. However, as effective treatment for restenosis caused by stent fracture is not yet established, stent-less PCI may be better in these types of lesions. We enrolled and analyzed lesions unsuitable for metal stenting based on 4 reasons described previously. However, lesion morphology may differ among inclusion criteria. For example, the reference diameter may differ between patients with very small coronary vessel and those who have surgery planned.

In conclusion, the role of DCB in de novo CAD still remains unclear in the era of improved and new-generation DES. However, for lesions that are unsuitable for metal stents and patients with a high bleeding risk, stent-less PCI using a DCB may be preferable. The present study also highlighted that optimal lesion preparation is key to a successful DCB strategy. Further supportive clinical data are needed to determine $\mathrm{DCB}$ criteria for CAD patients.

\section{References}

1. Kastrati A, Mehilli J, Pache J, Kaiser C, Valgimigli M, Kelbaek $\mathrm{H}$, et al. Analysis of 14 trials comparing sirolimus-eluting stents with bare-metal stents. N Engl J Med 2007; 356: 1030-1039.

2. Raisuke I, Yuji I, Akiyoshi M, Hiroyoshi N, Kazuhiro H. Predictors of restenosis after implantation of $2.5 \mathrm{~mm}$ stents in small coronary arteries. Circ J 2004; 68: 236-240.

3. Chung WS, Park CS, Seung KB, Kim PJ, Lee JM, Koo BK, et al. The incidence and clinical impact of stent strut fractures developed after drug-eluting stent implantation. Int J Cardiol 2008; 125: 325-331.

4. Enomoto Y, Ijijima R, Tokue M, Ito N, Nagashima Y, Araki T, et al. Bleeding risk with triple antithrombotic therapy in patients with atrial fibrillation and drug-eluting stents. Cardiovasc Interv Ther 2014; 29: 193-199.

5. Jackson D, Tong D, Layland J. A review of the coronary applications of the drug coated balloon. Int J Cardiol 2017; 226: $77-86$.

6. Harada Y, Colleran R, Pinieck S, Giacoppo D, Michel J, Kufner $\mathrm{S}$, et al. Angiographic and clinical outcomes of patients treated with drug-coated balloon angioplasty for in-stent restenosis after coronary bifurcation stenting with a two-stent technique. EuroIntervention 2017; 12: 2132-2139.

7. Kleber FX, Schulz A, Waliszewski M, Hauschild T, Böhm M, Dietz U, et al. Local paclitaxel induces late lumen enlargement in coronary arteries after balloon angioplasty. Clin Res Cardiol 2015; 104: $217-225$.

8. Taguchi I, Kageyama M, Kanaya T, Abe S, Node K, Inoue T. Clinical significance of non-slip element balloon angioplasty 
for patients of coronary artery disease: A preliminary report. $J$ Cardiol 2014; 63: 19-23.

9. Cortese B, Silva Orrego P, Agostoni P, Buccheri D, Piraino D, Andolina $G$, et al. Effect of drug-coated balloons in native coronary artery disease left with a dissection. JACC Cardiovasc Interv 2015; 8: 2003-2009.

10. Koeln PJ, Scheller B, Liew HB, Rissanen TT, Ahmad WA, Weser $\mathrm{R}$, et al. Treatment of chronic total occlusions in native coronary arteries by drug-coated balloons without stenting: A feasibility and safety study. Int J Cardiol 2016; 225: 262-267.

11. Cutlip DE, Windecker S, Mehran R, Boam A, Cohen DJ, van Es $\mathrm{GA}$, et al. Clinical end points in coronary stent trials: A case for standardized definitions. Circulation 2007; 115: 2344-2351.

12. Nakamura M, Muramatsu T, Yokoi H, Okada H, Ochiai M, Suwa S, et al. Outcomes of the largest multi-center trial stratified by the presence of diabetes mellitus comparing sirolimus-eluting stents (SES) and paclitaxel-eluting stents (PES) in patients with coronary artery disease. The Japan Drug-Eluting Stents Evaluation: A Randomized Trial (J-DESsERT). Cardiovasc Interv Ther 2015; 30: 103-114.

13. Ohya M, Kadota K, Kubo S, Tada T, Habara S, Shimada T, et al. Incidence, predictive factors, and clinical impact of stent recoil in stent fracture lesion after drug-eluting stent implantation. Int $J$ Cardiol 2016; 214: 123-129.

14. Ino Y, Kubo T, Kitabata H, Shimamura K, Shiono Y, Orii M, et al. Impact of hinge motion on in-stent restenosis after sirolimuseluting stent implantation. Circ J 2011; 75: 1878-1884.

15. Kimura $T$, Isshiki $T$, Hayashi $Y$, Oshima $S$, Namura $M$, Nakashima H, et al. Incidence and outcome of surgical procedures after sirolimus-eluting stent implantation: A report from the j-Cypher registry. Cardiovasc Interv Ther 2010; 25: 29-39.

16. Iijima R, Tsunoda T, Yamamoto M, Shiba M, Wada M, Tsuji $\mathrm{T}$, et al. Fate of unprotected side branches as related to embolic complications during stent implantation for acute coronary syndromes using a distal protection procedure. Am J Cardiol 2005; 95: 636-639.

17. Iijima R, Nakamura M, Matsuyama Y, Muramatsu T, Yokoi H, Hara $\mathrm{H}$, et al. Effect of optimal medical therapy before procedures on outcomes in coronary patients treated with drug-eluting stents. Am J Cardiol 2016; 118: 790-796.

18. Morice MC, Urban P, Greene S, Schuler G, Chevalier B. Why are we still using coronary bare-metal stents? J Am Coll Cardiol 2013; 61: 1122-1123.

19. Saito S, Valdes-Chavarri M, Richardt G, Moreno R, Iniguez Romo A, Barbato E, et al. A randomized, prospective, intercontinental evaluation of a bioresorbable polymer sirolimus-eluting coronary stent system: The CENTURY II (Clinical Evaluation of New Terumo Drug-Eluting Coronary Stent System in the Treatment of Patients with Coronary Artery Disease) trial. Eur Heart J 2014; 35: 2021-2031.

20. Kandzari DE, Mauri L, Koolen JJ, Massaro JM, Doros G, Garcia-Garcia HM, et al. Ultrathin, bioresorbable polymer sirolimus-eluting stents versus thin, durable polymer everolimuseluting stents in patients undergoing coronary revascularisation (BIOFLOW V): A randomised trial. Lancet 2017; 390: 1843-1852.

21. Chakravarty T, White AJ, Buch M, Naik H, Doctor N, Schapira $\mathbf{J}$, et al. Meta-analysis of incidence, clinical characteristics and implications of stent fracture. Am J Cardiol 2010; 106: 1075-1080.

22. Kuramitsu S, Iwabuchi M, Haraguchi T, Domei T, Nagae A, Hyodo M, et al. Incidence and clinical impact of stent fracture after everolimus-eluting stent implantation. Circ Cardiovasc Interv 2012; 5: 663-671.

23. Frab A, Virmani R, Atkinson JB, Kolodgie FD. Plaque morphology and pathologic changes in arteries from patients dying after coronary balloon angioplasty. $\mathrm{J} \mathrm{Am}$ Coll Cardiol 1990; 16: $1421-1429$.

24. Honye J, Mahon DJ, Jain A, White CJ, Ramee SR, Wallis JB, et al. Morphological effects of coronary balloon angioplasty in vivo assessed by intravascular ultrasound imaging. Circulation 1992; 85: $1012-1025$.

25. Nakamura M, Isshiki T, Kimura T, Ogawa H, Yokoi H, Nanto $\mathrm{S}$, et al. Optimal cutoff value of $\mathrm{P} 2 \mathrm{Y} 12$ reaction units to prevent major adverse cardiovascular events in the acute periprocedural period: Post-hoc analysis of the randomized PRASFIT-ACS study. Int J Cardiol 2015; 182: 541-548.

26. Shiomi H, Morimoto T, Kitaguchi S, Nakagawa Y, Ishii K, Haruna Y, et al. The ReACT Trial: Randomized Evaluation of Routine Follow-up Coronary Angiography After Percutaneous Coronary Intervention Trial. JACC Cardiovasc Interv 2017; 10: $109-117$.

\section{Supplementary Files}

Supplementary File 1

Table S1. Baseline characteristics of 115 patients treated with drug-coated balloon: comparison of patients with and without TLR

Table S2. Baseline lesional and procedural characteristics of 115 patients treated with drug-coated balloon: comparison of patients with and without TLR

Please find supplementary file(s);

http://dx.doi.org/10.1253/circj.CJ-18-0121 